PROCEEDINGS OF THE

AMERICAN MATHEMATICAL SOCIETY

Volume 134, Number 11, November 2006, Pages 3309-3317

S 0002-9939(06)08377-8

Article electronically published on May 12, 2006

\title{
THE REDUCED MINIMUM MODULUS OF DRAZIN INVERSES OF LINEAR OPERATORS ON HILBERT SPACES
}

\author{
CHUN-YUAN DENG AND HONG-KE DU
}

(Communicated by Joseph A. Ball)

\begin{abstract}
In this article, we study the reduced minimum modulus of the Drazin inverse of an operator on a Hilbert space and give lower and upper bounds of the reduced minimum modulus of an operator and its Drazin inverse, respectively. Using these results, we obtain a characterization of the continuity of Drazin inverses of operators on a Hilbert space.
\end{abstract}

\section{INTRODUCTION}

Let $\mathcal{H}$ be a complex Hilbert space. Denote by $\mathcal{B}(\mathcal{H})$ the Banach space of all bounded linear operators on $\mathcal{H}$. For an operator $T \in \mathcal{B}(\mathcal{H})$, the symbols $\mathcal{N}(T)$ and $\mathcal{R}(T)$ will denote the null space and the range space of $T$, respectively. The spectrum of $T$ is denoted by $\sigma(T)$. Let $S_{\mathcal{M}}=\{x \in \mathcal{M}:\|x\|=1\}$ if $\mathcal{M}$ is a subspace of $\mathcal{H}$ and let $\overline{\mathcal{K}}$ be the closure of a subset $\mathcal{K}$ of $\mathcal{H}$. For $T \in \mathcal{B}(\mathcal{H})$, if there exists an operator $T^{D} \in \mathcal{B}(\mathcal{H})$ satisfying the three operator equations

$$
T T^{D}=T^{D} T, \quad T^{D} T T^{D}=T^{D}, \quad T^{k+1} T^{D}=T^{k},
$$

then $T^{D}$ is called a Drazin inverse of $T$. For $T \in \mathcal{B}(\mathcal{H})$, if its Drazin inverse exists, then the Drazin inverse $T^{D}$ of $T$ is unique ([1]). The eigenprojection $T^{\pi}=I-$ $T^{D} T$ of $T$ corresponding to the eigenvalue 0 is the uniquely determined idempotent operator with

$$
\mathcal{R}\left(T T^{D}\right)=\mathcal{R}\left(T^{k}\right)=\mathcal{R}\left(T^{D}\right)=\mathcal{N}\left(T^{\pi}\right) \quad \text { and } \quad \mathcal{N}\left(T T^{D}\right)=\mathcal{R}\left(T^{\pi}\right) .
$$

Recall that $\operatorname{asc}(T)(\operatorname{des}(T))$, the ascent (descent) of $T \in \mathcal{B}(\mathcal{H})$, is the smallest non-negative integer $n$ such that $\mathcal{N}\left(T^{n}\right)=\mathcal{N}\left(T^{n+1}\right)\left(\mathcal{R}\left(T^{n}\right)=\mathcal{R}\left(T^{n+1}\right)\right)$, that is, $\operatorname{asc}(T):=\min \left\{k: \mathcal{N}\left(T^{k}\right)=\mathcal{N}\left(T^{k+1}\right)\right\}\left(\operatorname{des}(T):=\min \left\{k: \mathcal{R}\left(T^{k}\right)=\mathcal{R}\left(T^{k+1}\right)\right\}\right)$. If no such $n$ exists, then $\operatorname{asc}(T)=\infty(\operatorname{des}(T)=\infty)$. It is well known that $\operatorname{des}(T)=$ $\operatorname{asc}(T)$ if $\operatorname{asc}(T)$ and $\operatorname{des}(T)$ are finite $([9,[12)$. An operator $T \in \mathcal{B}(\mathcal{H})$ has the Drazin inverse $T^{D}$ if and only if $T$ has finite ascent and descent, which is equivalent with the fact that 0 is a finite order pole of the resolvent operator $R_{\lambda}(T)=$ $(\lambda I-T)^{-1}$, say of order $k$. In such a case, $\operatorname{ind}(T)=\operatorname{asc}(T)=\operatorname{des}(T)=k$, and 0 is not the accumulated point of $\sigma(T)([6])$. If $\operatorname{dim} \mathcal{H}$, the dimension of $\mathcal{H}$, is finite,

Received by the editors May 11, 2005 and, in revised form, May 31, 2005.

2000 Mathematics Subject Classification. Primary 47A05, 46C07, 15A09.

Key words and phrases. Drazin inverse, reduced minimum modulus, gap between two subspaces.

This research was partially supported by the National Natural Science Foundation of China (10571113). 
then, for $T \in \mathcal{B}(\mathcal{H})$, it is clear that $\operatorname{asc}(T)$ and $\operatorname{des}(T)$ are finite. So all of the operators in $\mathcal{B}(\mathcal{H})$ are Drazin invertible when $\operatorname{dim} \mathcal{H}$ is finite. But if $\operatorname{dim} \mathcal{H}$ is infinite, then there exist operators which are not Drazin invertible. For example, let $S$ be a unilateral shift in $\mathcal{B}(\mathcal{H})$. Since $\mathcal{R}\left(S^{k+1}\right) \subseteq \mathcal{R}\left(S^{k}\right)$ and $\mathcal{R}\left(S^{k+1}\right) \neq \mathcal{R}\left(S^{k}\right)$ for each positive integer $n, \operatorname{des}(S)=\infty$. Thus $S$ is not Drazin invertible. Some details about Drazin inverses of operators appear in [1, [2, 4], 6], 7], 10]-12] and [14-[15].

The reduced minimum modulus $\gamma(T)$ of $T$ (see [8]) is defined by

$$
\gamma(T)=\inf \{\|T x\|: \operatorname{dist}(x, \mathcal{N}(T))=1\} .
$$

Thus, from the definition of $\gamma(T)$, we deduce that $\|T x\| \geq \gamma(T) \operatorname{dist}(x, \mathcal{N}(T))$ for any $x \in \mathcal{H}$. It is well known that $\mathcal{R}(T)$ is closed if and only if $\gamma(T)>0$. In this case, $\gamma(T)=\left\|T^{+}\right\|^{-1}$, where $T^{+}$is the Moore-Penrose inverse of $T$, is the unique solution of the four operator equations

$$
T X T=T, \quad X T X=X, \quad T X=(T X)^{*}, \quad X T=(X T)^{*}
$$

if there exists the Moore-Penrose inverse of $T$.

Let $\mathcal{M}$ and $\mathcal{N}$ be two closed linear subspaces of a Hilbert space $\mathcal{H}$. The gap between $\mathcal{M}$ and $\mathcal{N}$ (see [5, [8]) is defined by

$$
\operatorname{gap}(\mathcal{M}, \mathcal{N})=\max \{\delta(\mathcal{M}, \mathcal{N}), \delta(\mathcal{N}, \mathcal{M})\},
$$

where $\delta(\mathcal{M}, \mathcal{N})=\sup \{\operatorname{dist}(x, \mathcal{N}): x \in \mathcal{M},\|x\| \leq 1\}$.

In this note, we study the reduced minimum modulus $\gamma(T)$ of an operator $T \in$ $\mathcal{B}(\mathcal{H})$ and the reduced minimum modulus $\gamma\left(T^{D}\right)$ of its Drazin inverse $T^{D}$, and give out their lower and upper bounds. Simultaneously, we obtain a characterization of the continuity of Drazin inverses.

\section{Main RESUlts AND PROOFS}

As a motivation of this paper, we observe that there are many differences among the inverse, the Moore-Penrose inverse and the Drazin inverse of operators. In this section, we begin with some examples to show these differences. Let $T \in \mathcal{B}(\mathcal{H})$. It is known that $T$ has the Moore-Penrose inverse $T^{+}$if and only if $\mathcal{R}(T)$ is closed (that is, $\gamma(T)>0$ ). But the operator $T$ is not necessary to have the closed range if its Drazin inverse $T^{D}$ exists. The following example can illustrate it.

Example 1. Let a linear operator $T$ on the Hilbert space $\mathcal{H} \oplus \mathcal{H} \oplus \mathcal{H}$ with $\operatorname{dim} \mathcal{H}=$ $\infty$ be given by the $3 \times 3$ operator matrices,

$$
T=\left(\begin{array}{ccc}
A & 0 & 0 \\
0 & 0 & S \\
0 & 0 & 0
\end{array}\right),
$$

where $A \in \mathcal{B}(\mathcal{H})$ is invertible and $S \in \mathcal{B}(\mathcal{H})$ is an arbitrary operator with $\mathcal{R}(S)$ not being closed. As we know, there exist many operators in $\mathcal{B}(\mathcal{H})$ with non- closed ranges. It is easy to verify that $T^{D}$ exists and

$$
T^{D}=\left(\begin{array}{ccc}
A^{-1} & 0 & 0 \\
0 & 0 & 0 \\
0 & 0 & 0
\end{array}\right) .
$$

But $\mathcal{R}(T)=\mathcal{H} \oplus \mathcal{R}(S) \oplus 0$ is not closed. 
If $T \in \mathcal{B}(\mathcal{H})$ is invertible and $T_{n} \rightarrow T$ in norm as $n \rightarrow \infty$, then $T_{n}^{-1}$ exists for large enough $n$ and $T_{n}^{-1} \rightarrow T^{-1}$ as $n \rightarrow \infty$. The analogue is not available for the Drazin inverse.

Example 2. Let $A \in \mathcal{B}(\mathcal{H})$ be an invertible operator and let $S$ be the unilateral shift operator defied by $S e_{l}=e_{l+1}$, where $\left\{e_{l}, l=1,2,3, \ldots\right\}$ is an orthonormal basis for $\mathcal{H}$. Let $T_{n}$ and $T$ on the Hilbert space $\mathcal{H} \oplus \mathcal{H}$ be given by the $2 \times 2$ operator matrices

$$
T_{n}=\left(\begin{array}{cc}
A & 0 \\
0 & \frac{1}{n} S
\end{array}\right) \text { and } T=\left(\begin{array}{cc}
A & 0 \\
0 & 0
\end{array}\right) .
$$

Then $T_{n} \rightarrow T$ in norm as $n \rightarrow \infty, T^{D}$ exists and

$$
T^{D}=\left(\begin{array}{cc}
A^{-1} & 0 \\
0 & 0
\end{array}\right) \text {. }
$$

But, from $\mathcal{R}\left(T_{n}\right)=\mathcal{R}(A) \oplus \mathcal{R}(S), \mathcal{R}\left(T_{n}^{k}\right)=\mathcal{R}\left(A^{k}\right) \oplus \mathcal{R}\left(S^{k}\right)=\mathcal{R}(A) \oplus \mathcal{R}\left(S^{k}\right)$, $\mathcal{R}\left(S^{k+1}\right) \subseteq \mathcal{R}\left(S^{k}\right)$ and $\mathcal{R}\left(S^{k+1}\right) \neq \mathcal{R}\left(S^{k}\right)$, we know $\mathcal{R}\left(T_{n}^{k+1}\right) \subseteq \mathcal{R}\left(T_{n}^{k}\right)$ and $\mathcal{R}\left(T_{n}^{k+1}\right) \neq \mathcal{R}\left(T_{n}^{k}\right)$ for all $n=1,2,3, \ldots$, so $\operatorname{des}(S)=\infty$. Hence $T_{n}^{D}$ does not exist for all $n=1,2,3, \ldots$.

Although $T_{n}^{D}$ and $T^{D}$ exist for all $n, T_{n} \rightarrow T$ as $n \rightarrow \infty$ does not imply that $T_{n}^{D} \rightarrow T^{D}$ as $n \rightarrow \infty$.

Example 3. Let us consider the operator matrices

$$
T_{n}=\left(\begin{array}{ccc}
\frac{1}{n} I & 0 & 0 \\
0 & 0 & I \\
0 & 0 & 0
\end{array}\right) \text { and } T=\left(\begin{array}{ccc}
0 & 0 & 0 \\
0 & 0 & I \\
0 & 0 & 0
\end{array}\right)
$$

on the Hilbert space $\mathcal{H} \oplus \mathcal{H} \oplus \mathcal{H}$. Then $T_{n}$ and $T$ are not invertible and $T_{n} \rightarrow T$ in norm for large enough $n, T_{n}^{D}$ and $T^{D}$ exist and

$$
T_{n}^{D}=\left(\begin{array}{ccc}
n I & 0 & 0 \\
0 & 0 & 0 \\
0 & 0 & 0
\end{array}\right) \quad \text { and } \quad T^{D}=\left(\begin{array}{ccc}
0 & 0 & 0 \\
0 & 0 & 0 \\
0 & 0 & 0
\end{array}\right) .
$$

But $T_{n}^{D}$ does not converge to $T^{D}$ as $n \rightarrow \infty$.

Next, we will give some lemmas which will be useful later.

Lemma 1. For an operator $T \in \mathcal{B}(\mathcal{H}), \mathcal{R}(T)$ is closed if and only if there exists $X \in \mathcal{B}(\mathcal{H})$ such that $T X T=T$.

Lemma 2. Let $T \in \mathcal{B}(\mathcal{H})$ with the Drazin inverse $T^{D}$ and ind $(T)=k$. Then $\mathcal{R}\left(T^{k}\right)$ is closed and $\mathcal{R}\left(T^{D}\right)=\mathcal{R}\left(T^{k}\right)$.

Proof. From $T^{D} T T^{D}=T^{D}$ and $T T^{D}=T^{D} T$, we have $T^{D}=T^{k}\left(T^{D}\right)^{k+1}$. So $\mathcal{R}\left(T^{D}\right) \subseteq \mathcal{R}\left(T^{k}\right)$. On the other hand, from $T T^{D}=T^{D} T$ and $T^{k+1} T^{D}=T^{k}$, we can get $T^{k}=T^{D} T^{k+1}$ and $\mathcal{R}\left(T^{k}\right) \subseteq \mathcal{R}\left(T^{D}\right)$. Therefore, $\mathcal{R}\left(T^{D}\right)=\mathcal{R}\left(T^{k}\right)$.

Since $T^{D}$ is the Drazin inverse of $T, T^{D} T T^{D}=T^{D}$. By $\operatorname{Lemma} 1, \mathcal{R}\left(T^{D}\right)$ is closed.

The following result gives a characterization of the spectral radius $r_{\sigma}\left(T^{D}\right)$ of the Drazin inverse $T^{D}$ of $T$.

Lemma $3\left([6)\right.$. Let $T \in \mathcal{B}(\mathcal{H})$ with the Drazin inverse $T^{D}$ and ind $(T)=k$. Then $\operatorname{dist}(0, \sigma(T) \backslash\{0\})=\left(r_{\sigma}\left(T^{D}\right)\right)^{-1}$, where $r_{\sigma}(A)$ denotes the spectral radius of $A$. 
Proof. Since $T$ has the Drazin inverse $T^{D}$, then $\mathcal{R}\left(T^{k}\right)=\mathcal{R}\left(T^{D}\right)$ is closed. From $T T^{k}=T^{k+2} T^{D}=T^{k} T^{2} T^{D}, \mathcal{R}\left(T^{k}\right)$ is an invariant subspace of $T$. So $T$ and $T^{D}$ have the operator matrices

$$
T=\left(\begin{array}{cc}
T_{11} & T_{12} \\
0 & T_{22}
\end{array}\right) \text { and } T^{D}=\left(\begin{array}{cc}
T_{11}^{-1} & \sum_{i=0}^{k-1} T_{11}^{i-k-1} T_{12} T_{22}^{k-1-i} \\
0 & 0
\end{array}\right)
$$

with respect to the space decomposition $\mathcal{H}=\mathcal{R}\left(T^{k}\right) \oplus \mathcal{R}\left(T^{k}\right)^{\perp}$, where $T_{11}$ is invertible and $T_{22}$ is $k$-nilpotent (see [6], theorem 2.5). So $\sigma(T)=\sigma\left(T_{11}\right) \cup\{0\}$ and $\sigma\left(T^{D}\right)=\sigma\left(T_{11}^{-1}\right) \cup\{0\}$, since $\sigma\left(T_{22}\right)=\{0\}$ is a singleton. Hence

$$
\sigma\left(T^{D}\right)=\left\{\lambda^{+}: \lambda \in \sigma(T)\right\},
$$

where

$$
\lambda^{+}= \begin{cases}\lambda^{-1} & \text { if } \lambda \neq 0 \\ 0 & \text { if } \lambda=0\end{cases}
$$

By the definition of $\operatorname{dist}(x, \mathcal{M})$, it is easy to get

$$
\operatorname{dist}(0, \sigma(T) \backslash\{0\})=\left(r_{\sigma}\left(T^{D}\right)\right)^{-1} .
$$

For the reduced minimum modulus $\gamma\left(T^{D}\right)$ of $T^{D}$, we have the following estimation.

Theorem 4. Let $T \in \mathcal{B}(\mathcal{H})$ with the Drazin inverse $T^{D} \neq 0$ and $\operatorname{ind}(T)=k$. Then

$$
\frac{1}{\|T\|} \leq \gamma\left(T^{D}\right) \leq \frac{\left\|T^{D} T\right\|^{2}}{\left\|T^{D} T^{2}\right\|}
$$

Proof. For any $x \in \mathcal{H}, y \in \mathcal{N}\left(T^{D}\right)$, since $T T^{D}=T^{D} T$, we have

$$
\left\|T^{D} T(x)\right\|=\left\|T^{D} T(x-y)\right\| \leq\left\|T^{D} T\right\|\|x-y\|
$$

and

$$
\operatorname{dist}\left(x, \mathcal{N}\left(T^{D}\right)\right) \leq\left\|x-\left(I-T^{D} T\right) x\right\|=\left\|T^{D} T x\right\| .
$$

Thus

$$
\|T\|\left\|T^{D} x\right\| \geq\left\|T^{D} T x\right\| \geq \operatorname{dist}\left(x, \mathcal{N}\left(T^{D}\right)\right) \geq \frac{\left\|T^{D} T x\right\|}{\left\|T^{D} T\right\|} .
$$

Combining the definition of $\gamma(T)$ with (4), we have $\gamma\left(T^{D}\right) \geq\|T\|^{-1}$ and

$$
\left\|T^{D} x\right\| \geq \gamma\left(T^{D}\right) \operatorname{dist}\left(x, \mathcal{N}\left(T^{D}\right)\right) \geq \gamma\left(T^{D}\right) \frac{\left\|T^{D} T x\right\|}{\left\|T^{D} T\right\|} .
$$

Replacing $x$ by $T z, \forall z \in \mathcal{H}$ in (5), we have $\left\|T^{D} T z\right\| \geq \gamma\left(T^{D}\right) \frac{\left\|T^{D} T^{2} z\right\|}{\left\|T^{D} T\right\|}$, that is, $\gamma\left(T^{D}\right) \leq \frac{\left\|T^{D} T\right\|^{2}}{\left\|T^{D} T^{2}\right\|}$.

By the definition of the gap between two subspaces, we get the following results.

Theorem 5. Let $T \in \mathcal{B}(\mathcal{H})$ with the Drazin inverse $T^{D}$ and $\operatorname{ind}(T)=k$. If $\mathcal{N}(T) \neq$ $\mathcal{N}\left(T^{D}\right)$, then

$$
\delta\left(\overline{\mathcal{R}(T)}, \mathcal{R}\left(T^{D}\right)\right)=1, \delta\left(\mathcal{R}\left(T^{D}\right), \overline{\mathcal{R}(T)}\right)=0
$$

and

$$
\delta\left(\mathcal{N}\left(T^{D}\right), \mathcal{N}(T)\right)=1, \delta\left(\mathcal{N}(T), \mathcal{N}\left(T^{D}\right)\right)=0 .
$$


Proof. Since $T$ has the Drazin inverse $T^{D}$ and ind $(T)=k$, we have

$$
\mathcal{R}\left(T^{D}\right)=\mathcal{R}\left(T^{k}\right) \subseteq \mathcal{R}(T) \subseteq \overline{\mathcal{R}(T)} .
$$

Since $T^{D}=\left(T^{D}\right)^{2} T$ and $T^{k}=T^{k+1} T^{D}$,

$$
\mathcal{N}(T) \subseteq \mathcal{N}\left(T^{D}\right) \subseteq \mathcal{N}\left(T^{k}\right)
$$

If $\mathcal{N}(T) \neq \mathcal{N}\left(T^{D}\right)$, then $\operatorname{ind}(T)=k>1$ and $\mathcal{R}\left(T^{D}\right) \neq \mathcal{R}(T)$. Since $\mathcal{R}\left(T^{D}\right) \subseteq$ $\overline{\mathcal{R}(T)}$, by definition of the gap function $\delta$ between two subspaces, we have

$$
\delta\left(\mathcal{R}\left(T^{D}\right), \overline{\mathcal{R}(T)}\right)=0 .
$$

Since $\mathcal{R}\left(T^{D}\right) \neq \mathcal{R}(T)$, there exists $x \in \overline{\mathcal{R}(T)} \cap \mathcal{R}\left(T^{D}\right)^{\perp}$ with $\|x\|=1$ such that

$$
\operatorname{dist}\left(x, \mathcal{R}\left(T^{D}\right)\right)^{2}=\|x\|^{2}-\left\|P_{\mathcal{R}\left(T^{D}\right)} x\right\|^{2}=1 .
$$

Hence

$$
\delta\left(\overline{\mathcal{R}(T)}, \mathcal{R}\left(T^{D}\right)\right)=1
$$

By the similar argument, we can obtain formula (7). Hence the proof of this part is omitted.

Remark. (1) Theorem 3 gives us an example to show that, for two closed subspaces $\mathcal{M}$ and $\mathcal{N}, \delta(\mathcal{M}, \mathcal{N})$ is not symmetric, that is, in general $\delta(\mathcal{M}, \mathcal{N}) \neq \delta(\mathcal{N}, \mathcal{M})$. But $\delta(\mathcal{M}, \mathcal{N})=\delta\left(\mathcal{N}^{\perp}, \mathcal{M}^{\perp}\right)$ since $\operatorname{dist}(x, \mathcal{M})=\sup _{y \in S_{\mathcal{M}^{\perp}}}|\langle x, y\rangle|$ (see [8]). In fact,

$$
\begin{aligned}
\delta(\mathcal{M}, \mathcal{N}) & =\sup _{x \in S_{\mathcal{M}}} \operatorname{dist}(x, \mathcal{N}) \\
& =\sup _{x \in S_{\mathcal{M}}} \sup _{y \in S_{\mathcal{N}} \perp}|\langle x, y\rangle| \\
& =\sup _{y \in S_{\mathcal{N}^{\perp}}} \sup _{x \in S_{\mathcal{M}}}|\langle x, y\rangle| \\
& =\sup _{y \in S_{\mathcal{N}^{\perp}}} \operatorname{dist}\left(y, \mathcal{M}^{\perp}\right) \\
& =\delta\left(\mathcal{N}^{\perp}, \mathcal{M}^{\perp}\right) .
\end{aligned}
$$

(2) Let $T \in \mathcal{B}(\mathcal{H})$ with the Drazin inverse $T^{D}$ and ind $(T)=k$. If $T$ and $T^{D}$ have the operator matrices (3), respectively, and $x_{0}+x \in \mathcal{N}(T)$, where $x_{0} \in \mathcal{R}\left(T^{k}\right)$ and $x \in \mathcal{R}\left(T^{k}\right)^{\perp}$, then

$$
\left(\begin{array}{cc}
T_{11} & T_{12} \\
0 & T_{22}
\end{array}\right)\left(\begin{array}{l}
x_{0} \\
x
\end{array}\right)=0
$$

So we have

$$
\mathcal{N}(T)=\left\{\left(\begin{array}{c}
-T_{11}^{-1} T_{12} x \\
x
\end{array}\right): x \in \mathcal{N}\left(T_{22}\right)\right\} .
$$

Similarly, if $y_{0}+y \in \mathcal{N}\left(T^{D}\right)$, where $y_{0} \in \mathcal{R}\left(T^{k}\right)$ and $y \in \mathcal{R}\left(T^{k}\right)^{\perp}$, then

$$
\left(\begin{array}{cc}
T_{11}^{-1} & \sum_{i=0}^{k-1} T_{11}^{i-k-1} T_{12} T_{22}^{k-1-i} \\
0 & 0
\end{array}\right)\left(\begin{array}{l}
y_{0} \\
y
\end{array}\right)=0 .
$$

So we have

$$
\mathcal{N}\left(T^{D}\right)=\left\{\left(\begin{array}{c}
-\sum_{i=0}^{k-1} T_{11}^{i-k} T_{12} T_{22}^{k-1-i} y \\
y
\end{array}\right): y \in \mathcal{R}\left(T^{k}\right)^{\perp}\right\} .
$$

In general, $\mathcal{N}\left(T_{22}\right) \subseteq \mathcal{N}\left(T^{k}\right)^{\perp}$; this shows that $\mathcal{N}(T) \subseteq \mathcal{N}\left(T^{D}\right)$. Moreover, $\mathcal{N}(T)=$ $\mathcal{N}\left(T^{D}\right)$ if and only if $\mathcal{N}\left(T_{22}\right)=\mathcal{N}\left(T^{k}\right)^{\perp}$. In this case, ind $(T)=1$, that is, $\mathcal{N}\left(T_{22}\right)=$ $\mathcal{N}(T)^{\perp}$.

Theorem 6. Let $T \in \mathcal{B}(\mathcal{H})$ with the Drazin inverse $T^{D}$ and $\operatorname{ind}(T)=k$. If $\mathcal{N}(T) \neq$ $\mathcal{N}\left(T^{D}\right)$, then

$$
\gamma(T) \leq\left\|T-T^{D}\right\|
$$


Proof. If $\mathcal{R}(T)$ is not closed, then $\gamma(T)=0$. It is clear that $\gamma(T) \leq\left\|T-T^{D}\right\|$.

Next, we assume that $\mathcal{R}(T)$ is closed.

For any $z \in \mathcal{N}(T)$ and $u \in \mathcal{R}(T)$ with $\|u\|=1$, there exists $x \in \mathcal{H}$ such that $T x=u$ and

$$
\begin{aligned}
\operatorname{dist}\left(u, \mathcal{R}\left(T^{D}\right)\right) & \leq\left\|T x-T^{D}(x-z)\right\| \\
& =\left\|T(x-z)-T^{D}(x-z)\right\| \\
& \leq\left\|T-T^{D}\right\|\|x-z\| .
\end{aligned}
$$

So $\delta\left(\mathcal{R}(T), \mathcal{R}\left(T^{D}\right)\right) \leq\left\|T-T^{D}\right\|\|x-z\|$. Since $\|T x\| \geq \gamma(T) \operatorname{dist}(x, \mathcal{N}(T))$ and $\mathcal{R}(T)$ is closed,

$$
\operatorname{dist}(x, \mathcal{N}(T)) \leq \frac{\|T x\|}{\gamma(T)}=\frac{1}{\gamma(T)} .
$$

That is, $1=\delta\left(\mathcal{R}(T), \mathcal{R}\left(T^{D}\right)\right) \leq\left\|T-T^{D}\right\| \frac{1}{\gamma(T)}$ by Theorem 5 . The proof is completed.

The following theorem gives a relation of the reduced minimum modulus of the operators $T$ and $T^{D}$.

Theorem 7. Let $T \in \mathcal{B}(\mathcal{H})$ with the Drazin inverse $T^{D}$ and $\operatorname{ind}(T)=k$. Then

$$
\frac{1}{\left\|T^{k+1}\right\|}\left(\gamma(T)-\left\|T^{k}-T\right\|\right) \leq \gamma\left(T^{D}\right) \leq\left\|\left(T^{D}\right)^{k+1}\right\| \gamma\left(T^{k}\right) .
$$

Proof. From $T^{D} T T^{D}=T^{D}$ and $T T^{D}=T^{D} T$, we have $T^{D}=\left(T^{D}\right)^{k+1} T^{k}$. Equation (8) shows that $\mathcal{N}(T)^{\perp} \supseteq \mathcal{N}\left(T^{D}\right)^{\perp} \supseteq \mathcal{N}\left(T^{k}\right)^{\perp}$. Therefore, by the definition of $\gamma\left(T^{D}\right)$, we have

$$
\begin{aligned}
\gamma\left(T^{D}\right) & =\inf \left\{\left\|T^{D} x\right\|: \operatorname{dist}\left(x, \mathcal{N}\left(T^{D}\right)\right)=1\right\} \\
& =\inf \left\{\left\|T^{D} x\right\|: x \in S_{\left.\mathcal{N}\left(T^{D}\right)^{\perp}\right\}}\right. \\
& \leq \inf \left\{\left\|T^{D} x\right\|: x \in S_{\left.\mathcal{N}\left(T^{k}\right)^{\perp}\right\}}\right. \\
& =\inf \left\{\left\|\left(T^{D}\right)^{k+1} T^{k} x\right\|: x \in S_{\mathcal{N}\left(T^{k}\right)^{\perp}}\right\} \\
& \leq\left\|\left(T^{D}\right)^{k+1}\right\| \inf \left\{\left\|T^{k} x\right\|: x \in S_{\left.\mathcal{N}\left(T^{k}\right)^{\perp}\right\}}\right. \\
& \leq\left\|\left(T^{D}\right)^{k+1}\right\| \gamma\left(T^{k}\right) .
\end{aligned}
$$

On the other hand, observing that $T^{k}=T^{k+1} T^{D}$, we get

$$
\begin{aligned}
\gamma\left(T^{D}\right) & =\inf \left\{\left\|T^{D} x\right\|: x \in S_{\left.\mathcal{N}\left(T^{D}\right)^{\perp}\right\}}\right. \\
& \geq \frac{1}{\left\|T^{k+1}\right\|} \inf \left\{\left\|T^{k} x\right\|: x \in S_{\mathcal{N}\left(T^{D}\right)^{\perp}}\right\} \\
& \geq \pi T^{k+1} \| \inf \left\{\left\|T^{k} x\right\|: x \in S_{\mathcal{N}(T)^{\perp}}\right\} \\
& \geq \frac{1}{\left\|T^{k+1}\right\|} \inf \left\{\|T x\|-\left\|T^{k} x-T x\right\|: x \in S_{\mathcal{N}(T)^{\perp}}\right\} \\
& \geq \frac{1}{\left\|T^{k+1}\right\|}\left(\gamma(T)-\left\|T^{k}-T\right\|\right) .
\end{aligned}
$$

Hence

$$
\frac{1}{\left\|T^{k+1}\right\|}\left(\gamma(T)-\left\|T^{k}-T\right\|\right) \leq \gamma\left(T^{D}\right) \leq\left\|\left(T^{D}\right)^{k+1}\right\| \gamma\left(T^{k}\right)
$$

Combining the proceeding theorem with Theorem 4, we obtain the following result.

Corollary 8. Let $T \in \mathcal{B}(\mathcal{H})$ with the Drazin inverse $T^{D} \neq 0$ and $\operatorname{ind}(T)=k$. Then

$$
\gamma\left(T^{k}\right) \geq \frac{1}{\|T\|\left\|\left(T^{D}\right)^{k+1}\right\|} .
$$


Note that $\mathcal{N}\left(T^{D}\right)=\mathcal{N}\left(T^{D} T T^{D}\right) \supseteq \mathcal{N}\left(T T^{D}\right) \supseteq \mathcal{N}\left(T^{D}\right)$. So $\mathcal{N}\left(T^{D}\right)=\mathcal{N}\left(T T^{D}\right)$ and $\gamma\left(T T^{D}\right)=\inf \left\{\left\|T T^{D} x\right\|: x \in S_{\mathcal{N}\left(T T^{D}\right)^{\perp}}\right\} \leq\|T\| \inf \left\{\left\|T^{D} x\right\|: x \in S_{\mathcal{N}\left(T^{D}\right)^{\perp}}\right\}=$ $\|T\| \gamma\left(T^{D}\right)$. Thus we have the following corollary.

Corollary 9. Let $T \in \mathcal{B}(\mathcal{H})$ with the Drazin inverse $T^{D}$ and $\operatorname{ind}(T)=k$. Then

$$
\frac{\gamma\left(T T^{D}\right)}{\|T\|} \leq \gamma\left(T^{D}\right) \leq\left\|\left(T^{D}\right)^{k+1}\right\| \gamma\left(T^{k}\right)
$$

Let $\mathcal{M}$ and $\mathcal{N}$ be two closed subspaces of $\mathcal{H}$. If $P_{\mathcal{M}}$ and $P_{\mathcal{N}}$ are orthogonal projections onto $\mathcal{M}$ and $\mathcal{N}$, respectively, we have the following lemma for the gap between the two subspaces $\mathcal{M}$ and $\mathcal{N}$.

Lemma 10 ([5], 8]). Let $\mathcal{M}$ and $\mathcal{N}$ be two closed subspaces of $\mathcal{H}$. If $P_{\mathcal{M}}$ and $P_{\mathcal{N}}$ are orthogonal projections onto $\mathcal{M}$ and $\mathcal{N}$, respectively, then

$$
\begin{aligned}
\operatorname{gap}(\mathcal{M}, \mathcal{N}) & =\max \left\{\left\|P_{\mathcal{M}}\left(I-P_{\mathcal{N}}\right)\right\|,\left\|P_{\mathcal{N}}\left(I-P_{\mathcal{M}}\right)\right\|\right\} \\
& =\operatorname{gap}\left(\mathcal{M}^{\perp}, \mathcal{N}^{\perp}\right) \\
\left\|\left(I-P_{\mathcal{N}}\right) P_{\mathcal{M}}\right\| & \leq\left\|I-P_{\mathcal{N}}\right\|\left\|P_{\mathcal{M}}\right\| \operatorname{gap}\left(\mathcal{R}\left(P_{\mathcal{M}}\right), \mathcal{R}\left(P_{\mathcal{N}}\right)\right)
\end{aligned}
$$

and

$$
\left\|P_{\mathcal{N}}\left(I-P_{\mathcal{M}}\right)\right\| \leq\left\|P_{\mathcal{N}}\right\|\left\|I-P_{\mathcal{M}}\right\| \operatorname{gap}\left(\mathcal{N}\left(P_{\mathcal{M}}\right), \mathcal{N}\left(P_{\mathcal{N}}\right)\right) .
$$

Proof. (1) See corollary 7 in 5 .

(2) Let $y \in \mathcal{N}$. Then, for any $x \in \mathcal{H},\left(I-P_{\mathcal{N}}\right) P_{\mathcal{M}} x=\left(I-P_{\mathcal{N}}\right)\left(P_{\mathcal{M}} x-y\right)$, which implies

$$
\begin{aligned}
\left\|\left(I-P_{\mathcal{N}}\right) P_{\mathcal{M}} x\right\| & \leq\left\|\left(I-P_{\mathcal{N}}\right)\right\| \operatorname{dist}\left(P_{\mathcal{M}} x, \mathcal{N}\right) \\
& \leq\left\|\left(I-P_{\mathcal{N}}\right)\right\| \operatorname{gap}\left(\mathcal{R}\left(P_{\mathcal{M}}\right), \mathcal{R}\left(P_{\mathcal{N}}\right)\right)\left\|P_{\mathcal{M}} x\right\| \\
& \leq\left\|\left(I-P_{\mathcal{N}}\right)\right\|\left\|P_{\mathcal{M}}\right\| \operatorname{gap}\left(\mathcal{R}\left(P_{\mathcal{M}}\right), \mathcal{R}\left(P_{\mathcal{N}}\right)\right)\|x\| .
\end{aligned}
$$

Hence formula (9) holds.

Similarly, noting that $\mathcal{N}\left(P_{\mathcal{M}}\right)=\mathcal{R}\left(I-P_{\mathcal{M}}\right), \mathcal{N}\left(P_{\mathcal{N}}\right)=\mathcal{R}\left(I-P_{\mathcal{N}}\right)$, we can show

$$
\left\|P_{\mathcal{N}}\left(I-P_{\mathcal{M}}\right)\right\| \leq\left\|P_{\mathcal{N}}\right\|\left\|I-P_{\mathcal{M}}\right\| \operatorname{gap}\left(\mathcal{N}\left(P_{\mathcal{M}}\right), \mathcal{N}\left(P_{\mathcal{N}}\right)\right) .
$$

Remark. From the above proof, the inequalities (9) and (10) are also true when $P_{\mathcal{M}}$ and $P_{\mathcal{N}}$ are projections on $\mathcal{M}$ and $\mathcal{N}$, respectively, but not orthogonal projections.

To discuss the continuity of Drazin inverse in the rest of this paper, we need a lemma (see 8, I, Theorem 6.35).

Lemma 11 ([8]). Let $P^{\prime}, Q^{\prime}$ be two idempotents on $\mathcal{H}$ and let $\mathcal{M}=\mathcal{R}\left(P^{\prime}\right), \mathcal{N}=$ $\mathcal{R}\left(Q^{\prime}\right)$. Let $P, Q$ be the orthogonal projections on $\mathcal{M}, \mathcal{N}$, respectively. Then $\|P-Q\| \leq\left\|P^{\prime}-Q^{\prime}\right\|$.

In the corollary 1 of [11, Y. Wei and G. Chen give sufficient and necessary conditions for the continuity of Moore-Penrose inverses in a Hilbert space. With the application of the characterization of gap between two subspaces, we give a similar theorem for Drazin inverses.

Theorem 12. Let $T \in \mathcal{B}(\mathcal{H})$ with the Drazin inverse $T^{D}, T_{n} \rightarrow T$ as $n \rightarrow \infty$, and let $T_{n} \in \mathcal{B}(\mathcal{H})$ with Drazin inverse $T_{n}^{D}$ as $n$ large enough. Then the following statements are equivalent:

(1) $T_{n}^{D} \rightarrow T^{D}$ as $n \rightarrow \infty$. 
(2) $\operatorname{gap}\left(\mathcal{R}\left(T_{n}^{\pi}\right), \mathcal{R}\left(T^{\pi}\right)\right) \rightarrow 0$ and $\operatorname{gap}\left(\mathcal{N}\left(T_{n}^{\pi}\right), \mathcal{N}\left(T^{\pi}\right)\right) \rightarrow 0$ as $n$ large enough, where $T_{n}^{\pi}=I-T_{n}^{D} T_{n}$ and $T^{\pi}=I-T^{D} T$.

Proof. $(1) \Rightarrow(2)$ Let $O\left(T_{n}^{\pi}\right)$ and $O\left(T^{\pi}\right)$ be the orthogonal projections of $\mathcal{H}$ onto $\mathcal{R}\left(T_{n}^{\pi}\right)$ and $\mathcal{R}\left(T^{\pi}\right)$, respectively. By Lemma 11, we have

$$
\left\|O\left(T_{n}^{\pi}\right)-O\left(T^{\pi}\right)\right\| \leq\left\|T_{n}^{\pi}-T^{\pi}\right\|
$$

Since $T, T_{n}, T_{n}^{D} \in \mathcal{B}(\mathcal{H}), T_{n} \rightarrow T$ and $T_{n}^{D} \rightarrow T^{D}$ as $n \rightarrow \infty$, we have

$$
\left\|T_{n}^{\pi}-T^{\pi}\right\|=\left\|T_{n}^{D} T_{n}-T^{D} T\right\| \leq\left\|T_{n}\right\|\left\|T_{n}^{D}-T^{D}\right\|+\left\|T^{D}\right\|\left\|T_{n}-T\right\| \rightarrow 0
$$

as $n \rightarrow \infty$. Hence $O\left(T_{n}^{\pi}\right) \rightarrow O\left(T^{\pi}\right)$ as $n \rightarrow \infty$. From Lemma 10, we get

$$
\operatorname{gap}\left(\mathcal{R}\left(T_{n}^{\pi}\right), \mathcal{R}\left(T^{\pi}\right)\right)=\max \left\{\left\|O\left(T_{n}^{\pi}\right)\left(I-O\left(T^{\pi}\right)\right)\right\|,\left\|O\left(T^{\pi}\right)\left(I-O\left(T_{n}^{\pi}\right)\right)\right\|\right\} \rightarrow 0
$$

as $n \rightarrow \infty$. Since $O\left(T_{n}^{\pi *}\right) \rightarrow O\left(T^{\pi *}\right)$ as $n \rightarrow \infty$,

$$
\operatorname{gap}\left(\mathcal{N}\left(T_{n}^{\pi}\right), \mathcal{N}\left(T^{\pi}\right)\right)=\operatorname{gap}\left(\mathcal{R}\left(T_{n}^{\pi *}\right)^{\perp}, \mathcal{R}\left(T^{\pi *}\right)^{\perp}\right)=\operatorname{gap}\left(\mathcal{R}\left(T_{n}^{\pi *}\right), \mathcal{R}\left(T^{\pi *}\right)\right) \rightarrow 0
$$

as $n \rightarrow \infty$.

$(2) \Rightarrow(1)$ Since

$$
\left\|\left(I-T^{\pi}\right) T_{n}^{\pi}\right\| \leq\left\|I-T^{\pi}\right\|\left\|T_{n}^{\pi}\right\| \operatorname{gap}\left(\mathcal{R}\left(T_{n}^{\pi}\right), \mathcal{R}\left(T^{\pi}\right)\right) \rightarrow 0
$$

as $n \rightarrow \infty$ and

$$
\left\|T^{\pi}\left(I-T_{n}^{\pi}\right)\right\| \leq\left\|T^{\pi}\right\|\left\|I-T_{n}^{\pi}\right\| \operatorname{gap}\left(\mathcal{N}\left(T_{n}^{\pi}\right), \mathcal{N}\left(T^{\pi}\right)\right) \rightarrow 0
$$

as $n \rightarrow \infty$, we have

$$
\left\|T_{n}^{D} T_{n}-T T^{D}\right\|=\left\|T_{n}^{\pi}-T^{\pi}\right\| \leq\left\|T_{n}^{\pi}-T^{\pi} T_{n}^{\pi}\right\|+\left\|T^{\pi} T_{n}^{\pi}-T^{\pi}\right\| \rightarrow 0
$$

as $n \rightarrow \infty$. Therefore,

$$
\begin{aligned}
T_{n}^{D}-T^{D} & =T_{n}^{D} T T^{D}+T_{n}^{D}\left(I-T T^{D}\right)-\left(I-T_{n}^{D} T_{n}\right) T^{D}-T_{n}^{D} T_{n} T^{D} \\
& =T_{n}^{D}\left(T-T_{n}\right) T^{D}+T_{n}^{D}\left(I-T T^{D}\right)-\left(I-T_{n}^{D} T_{n}\right) T^{D} \\
& =T_{n}^{D}\left(T-T_{n}\right) T^{D}+T_{n}^{D}\left(T_{n}^{D} T_{n}-T T^{D}\right)-\left(T^{D} T-T_{n}^{D} T_{n}\right) T^{D} \\
& \rightarrow 0, \text { as } n \rightarrow \infty .
\end{aligned}
$$

Thus $T_{n}^{D} \rightarrow T^{D}$ in $\mathcal{B}(\mathcal{H})$.

Remark. (1) Example 2 shows that, in general, it does not imply that $T_{n}$ is Drazin invertible as $n$ large enough if $T_{n} \rightarrow T$ as $n \rightarrow \infty$ and $T$ is Drazin invertible. So in (1) of Theorem 12 the condition that $T_{n}$ has the Drazin inverse $T_{n}^{D}$ for $n$ large enough is necessary.

(2) Theorem 12 shows there is a close relation between the continuality of the Drazin inverse and the gaps of the ranges and the gaps of the null-spaces of the eigenprojections $T_{n}^{\pi}$ and $T^{\pi}$ of $T_{n}$ and $T$ corresponding to the eigenvalue 0 , respectively.

\section{ACKNOWLEDGEMENTS}

The authors would like to express their hearty thanks to the referee for his/her valuable comments and suggestions on the presentation of this paper. 


\section{REFERENCES}

[1] A. Ben-Israel, T. N. E. Greville, Generalized Inverses: Theory and Applications, 2nd Edition, Springer-Verlag, New York, 2003. MR1987382 (2004b:15008)

[2] S. L. Campbell, Recent applications of generalized inverses, Pitman, London, 1982. MR0666720 (83h:65010)

[3] J. Ding, L. J. Huang, Perturbation of Generalized Inverses of Linear Operators in Hilbert Spaces, Journal of Mathematical Analysis and Applications, 198(1996), 506-515. MR 1376277 (97i:47017)

[4] D. S. Djordjevic, Y. Wei, Additive results for the generalized Drazin inverse, J. Austral Math. Soc., 73(2002), 115-125. MR.1916312 (2003e:47002)

[5] H. Du, C. Deng, A new characterization of gaps between two subspaces, Proceedings of the American Mathematical Society, 133(2005), 3065-3070. MR2159786

[6] H. Du, C. Deng, The representation and characterization of Drazin inverses of operators on a Hilbert space, Linear Algebra and its Applications, 407(2005), 117-124. MR2161918 (2006d:47001)

[7] R. E. Hartwig, Gworong Wang, Yimin Wei, Some additive results on Drazin inverses, Linear Algebra and its Applications, 322(2001), 207-217. MR.1804521 (2001k:15004)

[8] D. T. Kato, Perturbation theory for linear operators, Springer, New York, 1966. MR0203473 $(34: 3324)$

[9] A. E. Taylar, D. C. Lay, Introduction to functional analysis 2nd Edition, John Wiley \& Sons, New York, Chichester, Brisbane, Toronto, 1980. MR0564653 (81b:46001)

[10] G. Wang, Y. Wei, S. Qiao, Generalized inverses: Theory and Computions, Science Press, Beijing, New York, 2004.

[11] Yimin Wei, Guoliang Chen, Perturbation of least squares problem in Hilbert spaces, Applied Mathematics and Computation, 121(2001), 177-183. MR.1830868(2002b:47022)

[12] Yimin Wei, Sanzheng Qiao, The representation and approximation of the Drazin inverse of a linear operator in Hilbert space, Applied Mathematics and Computation, 138(2003), 77-89. MR 1950346 (2003j:47001)

[13] Yimin Wei, A characterization and representation of the generalized inverse $A_{T, S}^{(2)}$ and its applications, Linear Algebra and its Applications, 280(1998), 87-96. MR1645022 (99g:15006)

[14] Yimin Wei, A characterization and representation of the Drazin inverse, SIAM J. Matrix Anal. Appl., 17(1996), 744-747. MR.1410699 (97k:15008)

[15] Liping Zhang, A characterization of the Drazin inverse, Linear Algebra and its Applications, 335(2001), 183-188. MR1850823 (2002f:15007)

[16] Chao Zhu, Jing Cai, Guo-liang Chen, Perturbation analysis for the reduced minimum modulus of bounded linear operator in Banach spaces, Applied Mathematics and Computation, 145(2003), 13-21. MR2005984 (2004g:47005)

College of Mathematics and Information Science, Shaanxi Normal University, Xi'an 710062, People's Republic of China

E-mail address: cy-deng@263.net

College of Mathematics and Information Science, Shaanxi Normal University, Xi'an 710062, People's Republic of China

E-mail address: hkdu@snnu.edu.cn 